\title{
A rare case of Interstitial lung disease complicated by overlapping syndrome with obstructive ventilatory dysfunction as the first symptom
}

\author{
Yuling LIANG", Yan LEI", Jiaqi XU, Liqi HUANG, Lian LIN, Yiqing ZHANG* \\ Department of Nephrology, Center of Nephrology and Urology, The Seventh Affiliated Hospital, Sun Yat-sen \\ University, Shenzhen, China \# Authors contributed equally to this paper \\ Correspondence: Yiqing ZHANG, Department of Nephrology, Center of Nephrology and Urology, the Seventh \\ Affiliated Hospital, Sun Yat-sen University, 628 Zhenyuan Rd., Guangming District., Shenzhen, Guangdong \\ Province, PR China.,518017,Email: zhangyq85@mail.sysu.edu.cn; https://orcid.org/0000-0001-7524-5895
}

Article History Received 5 September 2021; Accepted 25 October 2021; Published 31 December 2021

Cite this Article Yuling LIANG, Yan LEI, Jiaqi XU, Liqi HUANG, Lian LIN, Yiqing ZHANG. A rare case of

Interstitial lung disease complicated by overlapping syndrome with obstructive ventilatory dysfunction as the first

symptom [J].Medical Research, 2021.3(4):1-5, http://doi.org/10.6913/mr.0304.01

Copyright $@ 2021$ Creative Publishing Co., Limited.All rights reserved.Email:mrhk@mrhk.cc

\begin{abstract}
Interstitial lung disease is often a serious complication of connective tissue disease (CTD) , representing the first cause of death in CTD. However, there are few reports of SSc-RA overlap-associated interstitial pneumonia. Respiratory dysfunction as the first clinical manifestation is even rarely reported. We herein described a case of a male patient who developed significant respiratory dysfunction as the principal clinical sign for the past 6 months, plus newly developed skin thickening in bilateral upper limbs and pain and swelling of multiple joints for the past 6 weeks. Extensive immunological screening showed high titer of antinuclear antibodies(ANA), rheumatoid factor(RF), anti-cyclic peptide containing citrulline (anti-CCP), and positive anti-Scl-70 antibody. Chest high resolution computed tomography(HRCT) was performed and hence ILD was confirmed. Pulmonary function test (PFT) revealed obstructive ventilatory dysfunction rather than restrictive ventilatory dysfunction. So bronchodilation test was performed and asthma was considered. Finally, after a multidisciplinary team (MDT) discussion, the diagnosis of asthma and ILD associated with SSc-RA overlap was established. It is important to note that CTD associated pulmonary interstitial fibrosis must be considered as the differential diagnosis of any newly diagnosed interstitial pulmonary disease. In addition, if patient with dyspnea is highly suspicious of pulmonary interstitial fibrosis, bronchodilation test is still needed to exclude asthma in order to avoid misdiagnosis.
\end{abstract}

KEYWORDS Interstitial lung disease; Asthma; Systemic sclerosis; Rheumatoid arthritis; Overlap syndrome; case report

\section{Introduction}

ILD is defined as pulmonary fibrosis seen on HRCT or chest radiography, most pronounced in the basilar portions of the lungs, or the occurrence of idiopathic "Velcro" crackles on auscultation ${ }^{(1)}$. ILD may be secondary to connective tissue diseases, including rheumatoid arthritis (RA), systemic sclerosis (SSc), anti-neutrophil cytoplasmic antibody 
(ANCA) associated vasculitis (AAV) and systemic lupus erythematosus (SLE). Overlapping syndrome (OS) is defined as the co-existence of two or more independent connective tissue diseases (CTD). SSc patients may have similar clinical manifestation to that of RA patients, for example, joint pain. In clinical practice, SSc-RA overlap is less common. Only about 6\% of patients with SSc overlap with $\mathrm{RA}^{(2)}$. Only quite a few cases have reported lung involvement during SSc-RA overlap ${ }^{(3,4)}$. Lung involvement as the first clinical manifestation of SSc-RA overlap is even rarely reported. We herein discuss the case and review the associated literature of ILD and SSc-RA overlap.

\section{Case presentation}

A 55-year-old male electrician started to suffer from dry cough and dyspnoea 6 months ago. Symptoms often became worse at night. The symptoms gradually worsened and skin thickening in upper limbs and multiple joint pain and swelling newly developed 6 weeks prior to admission. He had no history of smoking or allergies. Family history of lung cancer (father) was noted.

Physical examination findings included clubbing fingers, skin thickening from bilateral fingers to elbows, swelling and tenderness at the proximal interphalangeal, metacarpophalangeal, wrist, shoulder, knee, and ankle joints. Coarse crepitations were heard bilaterally in both lung fields, worse at the base.

Pulmonary function test (PFT) was done which revealed obstructive ventilatory dysfunction with forced expiratory volume in 1 second (FEV1) of $2.13 \mathrm{~L}$ (76.5\% of the predicted), and $69.6 \%$ of forced vital capacity (FVC). The diffusion function was revealed normal with carbon monoxide diffusion capacity(DLCO) of $7.97 \mathrm{mmol} / \mathrm{min} / \mathrm{kpa}, 98.7 \%$ of the best. Bronchodilation test was showed positive (increase in FEV1 of $13.85 \%$ and $290 \mathrm{~mL}$ from baseline, 10-15 minutes after $400 \mathrm{mcg}$ albuterol). HRCT showed interstitial inflammation with fibrosis at the bottom of both lungs, characterized by honeycomb and grid-like changes in surrounding lung tissue, accompanied by partial bronchiectasis (Figure 1). Enlargement of lymph nodes in the intramediastinum, bronchial carina, lower esophageal space were also observed.

Transthoracic echocardiography (TTE), transesophageal endoscopic ultrasound-guided fine-needle aspiration (EUS-FNA) of mediastinal lymph nodes, electromyography (ECM), electronic gastroscope and positron emission tomography-computed tomography(PET-CT) were also performed but they provided no significant additional information.

The laboratory results showed an increased level of inflammatory parameters (erythrocyte sedimentation rate: ESR, $95 \mathrm{~mm} / \mathrm{h}$; C-reactive protein: CRP , 86.42 $\mathrm{mg} / \mathrm{dl})$, rheumatoid factor(RA)(188 units/ml), anti-cyclic citrullinated protein (anti-CCP) antibody(20.73 units/ml). Antinuclear antibodies (ANA) was positive, at titer 1:1000 with homogeneous pattern. Anti-Scl-70 antibody was strongly positive (+++). Blood gas analysis showed a drop in arterial partial pressure of oxygen (pH: 7.44; pO2: $61 \mathrm{mmHg}$; pCO2: 37 $\mathrm{mmHg})$.

After a multi-disciplinary team (MDT) discussion (pneumonologists, radiologists, and rheumatologists), the diagnosis was finally confirmed, that was asthma and ILD secondary to 
SSc-RA overlap syndrome. Then the patient was prescribed with methylprednisolone (40mg daily), tripterygium glycosides tablet (30mg daily) and budesonide and formoterol fumarate powder for inhalation. Dyspnea was significantly alleviated. Joint pain and tightness of the skin were also relieved. Prednisone tapered gradually as the above symptoms were improved. Up to now, followed up for more than one year, the clinical manifestations and the pulmonary function of the patient remain stable.

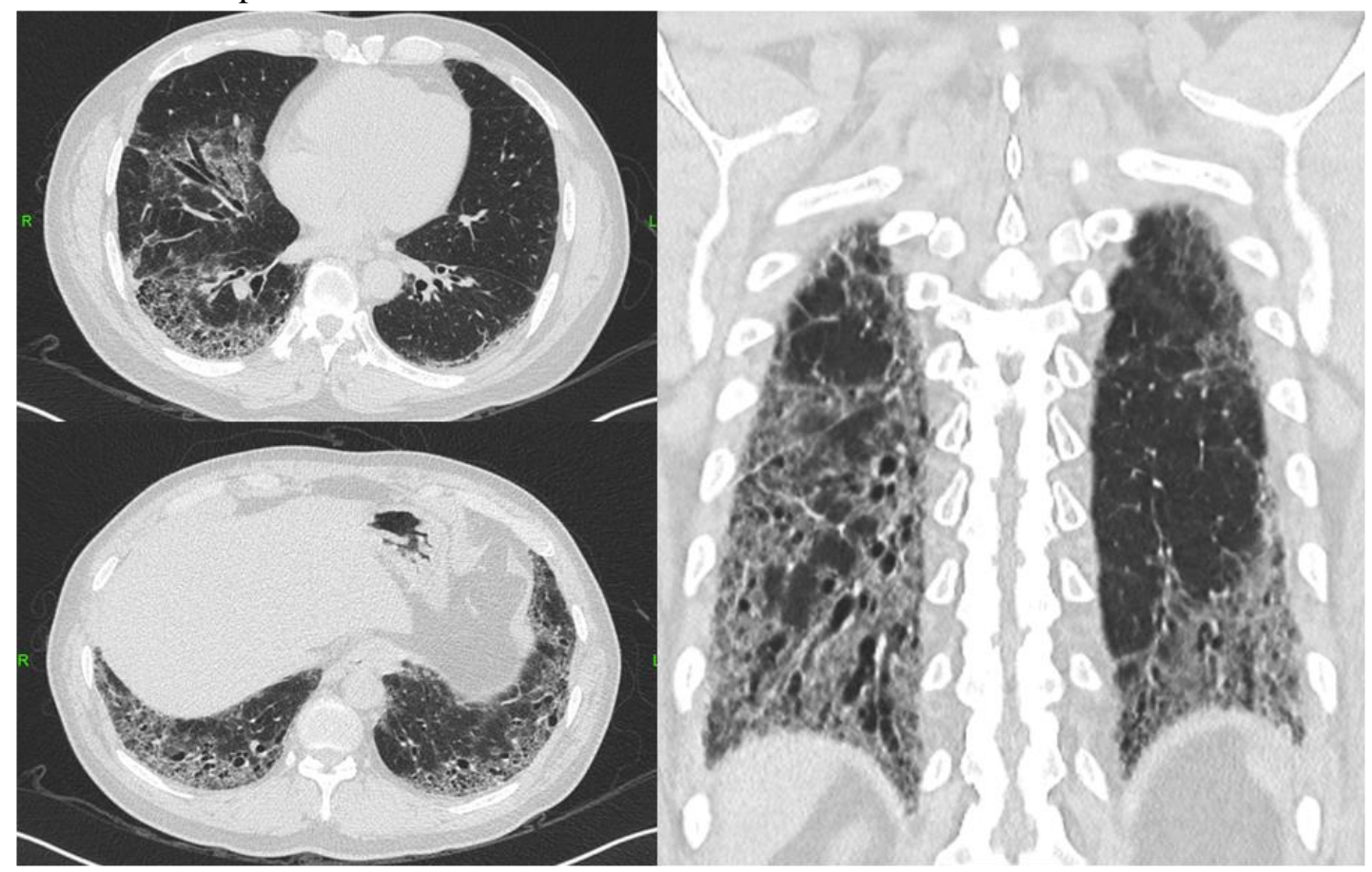

Figure 1 HRCT scan demonstrating ILD

\section{Discussion}

Systemic scleroderma (SSc) is an autoimmune disease characterized by vascular damage, autoimmunity, and tissue fibrosis ${ }^{(5)}$.HRCT imaging and pulmonary "Velcro" crackles were suggested of interstitial lung disease, and immunological test showed high titer of ANA and strongly posive anti-Scl-70 antibodies. The patient met the diagnostic criteria for systemic scleroderma(SSc) as defined by the American Society of Rheumatology in $2013^{(1)}$. Besides, the patient has more than ten joints involved, high-titer of RF and anti-CCP, increased CRP and ESR, and in addition, duration of symptoms was more than 6 weeks, which were consistent with the diagnosis of rheumatoid arthritis(RA) ${ }^{(6)}$. Therefore, the diagnosis of SSc-RA overlap syndrome(OS) was established. The respiratory symptoms and pulmonary function tests supported the diagnosis of asthma according to the Global Strategy for Asthma Management (GINA) ${ }^{(7)}$.

24-97\% of patients with SSc developed arthritis during the course of the disease $\mathrm{e}^{(5)}$. Anti-CCP antibodies and RF can be present in SSc patients, particularly in those with joint involvement, which may lead to misdiagnosis as RA on the early stage ${ }^{(2)}$. In fact, SSc-RA overlap is less common. The true rate of patients with SSc overlapped with RA is only $4.6 \%-6 \%(2,8)$. In a research of $61 \mathrm{SSc}$ patients, anti-CCP positive was observed only in patients with SSc-RA overlap ${ }^{(5)}$. So the detection of high titer anti-CCP antibodies in SSc may 
be helpful in determining the diagnosis of SSc-RA overlap syndrome and facilitating appropriate treatment.

ILD is particularly common in patients with SSc among connective tissue diseases. Data from the Spanish scleroderma study group showed that $43 \%$ of $1,374 \mathrm{SSc}$ patients showed pulmonary fibrosis on chest radiographs or HRCT(9). Indeed, ILD has been included in the SSc classification criteria. In SSc population, the presence of anti-Scl-70 antibodies, the decreased forced vital capacity (FVC) and the degree of fibrosis at baseline were predictors of progression of pulmonary fibrosis during follow-up ${ }^{(10)}$. The course of SSc-ILD was evident during the first 1-4 years of follow-up. Patients with no decline in lung function over four years had better results.

Rheumatoid arthritis also causes interstitial fibrosis alone. The reported prevalence of ILD in RA patients varies greatly, depending on the detection method and the study population selected. Studies have reported prevalence rates as low as about $4 \%$ to $68 \%^{(11,12)}$. Most lung disease occurs within 5 years after the initial diagnosis of $\mathrm{RA}^{(13)}$. The risk of developing ILD was higher in RA patients who were older at the time of disease onset, in male patients, and in individuals with high titers of RF and anti-CCP antibodies ${ }^{(14)}$.

Patients with overlap syndrome have a higher incidence of pulmonary interstitial fibrosis and a poorer prognosis ${ }^{(8)}$. Moreover, compared with RA patients, the frequency of HLA-DR3, HLA-DR7, HLA-DR11, and HLA-DRW53 increased significantly in SSc-RA patients. These suggested that SSC-RA overlap syndrome may be a unique genetic, immunological and clinical entity ${ }^{(8)}$.

PFT in cases with severe ILD secondry to SSc-RA overlap syndrome demonstrated restrictive pattern with reduced FEV1, and reduced $\mathrm{DLCO}^{(4)}$. However, in this case, PFT reported mild obstructive pulmonary ventilation dysfunction with normal diffusion function. Then bronchodilation test was performed, and the result proved positive. So the diagosis was finally confirmed as ILD complicated with asthma.

\section{Conclusion}

In this report, we presented a rare case of respiratory dysfunction caused by ILD (SSc-RA overlap syndrome-associated) and asthma. It is important to note that CTD associated pulmonary interstitial fibrosis must be considered as the differential diagnosis of any newly diagnosed interstitial pulmonary disease. A multidisciplinary approach with the intervention of respiratory physicians, radiologists and rheumatologists is recommended to achieve correct diagnosis. In addition, if patient with dyspnea is highly suspicious of pulmonary interstitial fibrosis but PFT report doesn't support the diagnosis of ILD, bronchodilation test is then needed to exclude asthma in order to avoid misdiagnosis.

\section{Declaration of conflict of interest}

The authors of this work do not declare any conflicts of interest.

\section{Acknowledgements}

We thank the multi-disciplinary team (pneumonologists, radiologists, and rheumatologists) in our hospital. 


\section{References}

1 Van den Hoogen F, Khanna D, Fransen J, Johnson SR, Baron M, Tyndall A, et al. 2013 classification criteria for systemic sclerosis: an American College of Rheumatology/European League against Rheumatism collaborative initiative. Arthritis Rheum. 2013;65(11):2737-47.

2 Wielosz E, Majdan M, Dryglewska M, Zwolak R. Anti-CCP antibodies and rheumatoid factor in systemic sclerosis: Prevalence and relationships with joint manifestations. Advances in clinical and experimental medicine : official organ Wroclaw Medical University. 2018;27(9):1253-7.

3 Yamakawa H, Hagiwara E, Yamanaka Y, Ikeda S, Sekine A, Kitamura H, et al. A 16-year Follow-up Case of Interstitial Pneumonia with Systemic Sclerosis-rheumatoid Arthritis Overlap Syndrome. Intern Med. 2017;56(12):1557-61.

4 Anand AS, Joseph PB, Vera-Vazquez E. A case of pulmonary fibrosis associated with rheumatoid arthritis, scleroderma sine scleroderma and ANCA associated vasculitis. SpringerPlus. 2014;3:513.

5 Horimoto AM, da Costa IP. Overlap between systemic sclerosis and rheumatoid arthritis: a distinct clinical entity? Rev Bras Reumatol Engl Ed. 2016;56(4):287-98.

6 Aletaha D, Neogi T, Silman AJ, Funovits J, Felson DT, Bingham CO, 3rd, et al. 2010 Rheumatoid arthritis classification criteria: an American College of Rheumatology/European League Against Rheumatism collaborative initiative. Arthritis Rheum. 2010;62(9):2569-81.

7 Global Initiative for Asthma. Global Strategy for Asthma Management and Prevention, 2021. Available from: www.ginasthma.org.

8 Szücs G, Szekanecz Z, Zilahi E, Kapitány A, Baráth S, Szamosi S, et al. Systemic sclerosis-rheumatoid arthritis overlap syndrome: a unique combination of features suggests a distinct genetic, serological and clinical entity. Rheumatology (Oxford, England). 2007;46(6):989-93.

9 Sánchez-Cano D, Ortego-Centeno N, Callejas JL, Fonollosa Plá V, Ríos-Fernández R, Tolosa-Vilella $\mathrm{C}$, et al. Interstitial lung disease in systemic sclerosis: data from the spanish scleroderma study group. Rheumatology international. 2018;38(3):363-74.

10 Hoffmann-Vold AM, Aaløkken TM, Lund MB, Garen T, Midtvedt $\varnothing$, Brunborg C, et al. Predictive value of serial high-resolution computed tomography analyses and concurrent lung function tests in systemic sclerosis. Arthritis \& rheumatology (Hoboken, NJ). 2015;67(8):2205-12.

11 Carmona L, González-Alvaro I, Balsa A, Angel Belmonte M, Tena X, Sanmartí R. Rheumatoid arthritis in Spain: occurrence of extra-articular manifestations and estimates of disease severity. Annals of the rheumatic diseases. 2003;62(9):897-900.

12 Mori S, Cho I, Koga Y, Sugimoto M. Comparison of pulmonary abnormalities on high-resolution computed tomography in patients with early versus longstanding rheumatoid arthritis. The Journal of rheumatology. 2008;35(8):1513-21.

13 Brown KK. Rheumatoid lung disease. Proceedings of the American Thoracic Society. 2007;4(5):443-8.

14 Bongartz T, Nannini C, Medina-Velasquez YF, Achenbach SJ, Crowson CS, Ryu JH, et al. Incidence and mortality of interstitial lung disease in rheumatoid arthritis: a population-based study. Arthritis and rheumatism. 2010;62(6):1583-91. 